\title{
Toll-like receptor agonist rMBP-NAP enhances antitumor cytokines production and CTL activity of peripheral blood mononuclear cells from patients with lung cancer
}

\author{
CONG DING $^{1 *}$, LI LI $^{1 *}$, YI ZHANG $^{2}$, ZHENYU JI $^{3}$, CHENGLONG ZHANG $^{1}$,

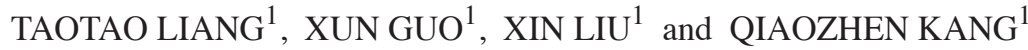

\begin{abstract}
${ }^{1}$ Department of Protein Function and Immunomodulatory Laboratory, School of Life Sciences, Zhengzhou University, Zhengzhou, Henan 450001; ${ }^{2}$ Department of Oncology, The First Affiliated Hospital of Zhengzhou University; ${ }^{3}$ Henan Academy of Medical and Pharmaceutical Sciences, Zhengzhou University, Zhengzhou, Henan 450052, P.R. China
\end{abstract}

Received December 8, 2017; Accepted May 30, 2018

DOI: $10.3892 / \mathrm{ol} .2018 .9182$

\begin{abstract}
Toll-like receptor (TLR) agonists are known for their ability to inhibit tumor progression via enhancing antitumor cytokines production and cytotoxic $\mathrm{T}$ lymphocyte (CTL) activity. Recombinant Helicobacter pylori neutrophil-activating protein fused with maltose-binding protein (rMBP-NAP) has been reported as a novel TLR agonist for antitumor treatment in murine models. The present study aimed to determine the potential and efficacy of the rMBP-NAP for antitumor treatment prior to further clinical trials. The rMBP-NAP was expressed and purified for subsequent experiments. Peripheral blood mononuclear cells (PBMCs) from health donors and patients with lung cancer (LC) were incubated with PBS and $0.2 \mathrm{mg} / \mathrm{ml} \mathrm{rMBP}-\mathrm{NAP}$. Antitumor cytokines production was assayed using ELISA and reverse transcription-quantitative polymerase chain reaction analysis. The cytolytic activity of PBMCs and the number of Interferon- $\gamma($ IFN- $\gamma)$-secreting cells were assayed using lactate dehydrogenase and Enzyme-linked ImmunoSpot assays, respectively. The results from the present study revealed that the expression of IFN- $\gamma$, interleukin (IL)-2, tumor necrosis factor- $\alpha$ and IL-12 of PBMCs from patients with LC and healthy donors were significantly increased following treatment with rMBP-NAP $(\mathrm{P}<0.05)$. Additionally, rMBP-NAP
\end{abstract}

Correspondence to: Professor Qiaozhen Kang or Dr Xin Liu, Department of Protein Function and Immunomodulatory Laboratory, School of Life Sciences, Zhengzhou University, 100 Science Road, Zhengzhou, Henan 450001, P.R. China

E-mail: qzkang@zzu.edu.cn

E-mail: liux@zzu.edu.cn

*Contributed equally

Key words: toll-like receptor agonists, recombinant Helicobacter pylori neutrophil-activating protein fused with maltose-binding protein, peripheral blood mononuclear cells, antitumor cytokines production, cytotoxic $\mathrm{T}$ lymphocyte activity significantly upregulated the number of IFN- $\gamma$-secreting cells in PBMCs and prominently increased the cytotoxic activity of PBMCs $(\mathrm{P}<0.05)$. Furthermore, the expression of TLR2 was significantly enhanced following rMBP-NAP stimulation $(\mathrm{P}<0.05)$, which indicated that rMBP-NAP may serve an antitumor role via TLR2 signaling pathways. Overall, these results demonstrated that rMBP-NAP possesses the potential to be a novel immunomodulatory candidate drug and requires further evaluation in clinical trials.

\section{Introduction}

Toll-like receptor (TLR) alone or combined with other antitumor drugs have been used in cancer therapy due to the capability of TLR agonists to enhance the immune response of patients with cancer by improving the function of innate and adaptive immune cells $(1,2)$. Currently, the US Food and Drug Administration (FDA) have licensed three TLRs agonists, including Bacille Calmette-Guérin (BCG), Monophosphoryl lipid A and Imiquimod, for use as prophylactics and/or therapeutic agents in cancer treatment (3-5). Although abundant natural and synthetic TLR agonists have been discovered and developed, a number of clinical research studies have reported that various TLR agonists that exhibited promising results in in vivo studies were not efficacious in humans $(6,7)$. The ineffectiveness of TLR agonists in clinical studies may be ascribed to two primary reasons: i) The deviation of immune cell phenotypes and the differential expression of the TLR gene between human and animals; and ii) the immune system in patients with cancer is compromised $(8,9)$. Therefore, comprehensive preclinical experiments are required to determine whether a novel TLR agonist may be an candidate antitumor drug.

Recombinant Helicobacter pylori neutrophil-activating protein fused with maltose-binding protein (rMBP-NAP), consisting Helicobacter pylori neutrophil activating protein fused with the Escherichia coli maltose binding protein, has been reported as a novel TLR agonist (10-12). In our previous research, local administration of rMBP-NAP inhibited tumor growth by activating TLR2 to trigger a significant Th1 type immune response in two murine models of hepatoma $\mathrm{H} 22$ 
and sarcoma S180 (13). Furthermore, following rMBP-NAP treatment in a B16-F10-induced metastatic lung cancer model, the survival rates were significant increased and the number of metastatic lung nodules were markedly reduced. During the treatment of the pulmonary metastasis, the release of antitumor cytokines, including interleukin (IL)-12, interferon- $\gamma$ (IFN- $\gamma$ ), IL-27, chemokine (C-C motif) ligand (CCL)2, CCL20, vascular cell adhesion molecule (VCAM)-1 and intercellular cell adhesion molecule (ICAM)-1, were also significantly increased (14). These previous studies indicate that rMBP-NAP may be a potential novel antitumor immunomodulatory drug for cancer treatment.

The present study further assessed the stimulation of the immune response by rMBP-NAP treatment on PBMCs from patients with lung cancer and healthy donors. The results suggested that rMBP-NAP promoted antitumor cytokine production, including the production of INF- $\gamma$, IL-2, TNF- $\alpha$ and IL-12, and enhanced the cluster of differentiation (CD) $4^{+}$ cytotoxic T lymphocyte (CTL) and CD $8^{+} \mathrm{CTL}$ cytotoxic effect. The present study further laid the groundwork for research into the efficacy of rMBP-NAP for clinical trials.

\section{Materials and methods}

Cell preparations. PBMCs from 12 health donors and 12 patients with lung cancer (LC) who had not previously received immunotherapy at the First Affiliated Hospital of Zhengzhou University (Zhengzhou, Henan, China) between April 2012 and October 2012 were isolated using Ficoll-Paque gradient centrifugation. Cells were centrifuged at $1,000 \mathrm{x}$ g for $25 \mathrm{~min}$ at $24^{\circ} \mathrm{C}$ and cultured in Iscove's modified Dulbecco's medium supplemented with $10 \%$ inactivated fetal calf serum (both from Thermo Fisher Scientific, Inc., Waltham, MA, USA). Written informed consent was obtained from all participants and the present study was approved by the Ethics Committee of the First Affiliated Hospital of Zhengzhou University (Zhengzhou, China).

Expression and purification of $r M B P-N A P$. The experimental procedures involved in the present study are all based on our previous published study (10). The rMBP-NAP was expressed by $E$. coli $\mathrm{TB} 1$ (pMAL-c2x-napA) at $37^{\circ} \mathrm{C}$, isopropyl $\beta$-D-1-thiogalactopyranoside was added and induced for $3 \mathrm{~h}$. The expression of rMBP-NAP was identified on $10 \%$ SDS-PAGE and was purified by amylose affinity chromatography. Endotoxin was removed with agarose bed columns containing immobilized polymyxin B (Thermo Fisher Scientific, Inc.).

Reverse transcription-quantitative polymerase chain reaction $(R T-q P C R)$. PBMCs from patients with $\mathrm{LC}$ and healthy donors were seed at a density of $2 \times 10^{6}$ cells/well and treated with rMBP-NAP $(0.2 \mathrm{mg} / \mathrm{ml})$ or PBS (as a control). The total RNA from the cultured PBMCs was extracted using TRIzol reagent (Life Technologies; Thermo Fisher Scientific, Inc.) and reverse transcribed into cDNA using the PrimerScript 1st Strand cDNA Synthesis kit (Takara Bio, Inc., Otsu, Japan). The thermocycling conditions maintained were as follows: $37^{\circ} \mathrm{C}$ for $15 \mathrm{~min} ; 85^{\circ} \mathrm{C}$ for $5 \mathrm{sec}$. RT-qPCR of the relative gene expression of TLR2 and IL-12p35 was performed using a SYBR Premix Ex TaqTMII (Takara Bio, Inc.) on an ABI 7500 Fast instrument (Applied Biosystems; Thermo Fisher Scientific, Inc.). The genes were amplified with the following primers: TLR2 forward, 5'-CCTGGCCCTCTC TACAAACTT-3' and reverse, 5'-ACTGTGTATTCGTGT GCTGGATA-3'; IL-12p35 forward, 5'-ATGGCCCTGTGC CTTAGTAGT-3' and reverse, 5'-CGGTTCTTCAAGGGA GGATTTT-3'; and GAPDH forward, 5'-TGATGACATCAA GAAGGTGG-3' and reverse, 5'-TTACTCCTTGGAGGC CATGT-3'. The thermocycling profile was as follows: $10 \mathrm{~min}$ at $95^{\circ} \mathrm{C}$ followed by 40 cycles of $15 \mathrm{sec}$ at $95^{\circ} \mathrm{C}$ and $1 \mathrm{~min}$ at $60^{\circ} \mathrm{C}$. Relative mRNA expression levels were calculated for each gene following normalization with GAPDH using the $2^{-\Delta \Delta \mathrm{Cq}}$ method (15).

EILSA. PBMCs from patients with LC and healthy donors were seeded at $2 \times 10^{6}$ cells/and treated with rMBP-NAP $(0.2 \mathrm{mg} / \mathrm{ml})$ or PBS (as a control) for 5 days. The cell culture supernatants were collected, and were used to detect the concentration of IFN- $\gamma$ (cat no. 570209), IL-2 (cat no. 431804) and TNF- $\alpha$ (cat no. 570109) secreted using ELISA kits (all from BioLegend, Inc., San Diego, CA, USA). The absorbance was measured at $450 \mathrm{~nm}$ using a SpectraMax absorbance reader (Molecular Devices, LLC, Sunnyvale, CA, USA).

Enzyme-linked ImmunoSpot (ELISPOT). PBMCs from patients with LC and healthy donors were seed at $2 \times 10^{6}$ cells in each well and treated with rMBP-NAP $(0.1$ or $0.2 \mathrm{mg} / \mathrm{ml})$ or PBS (as a control). The number of IFN- $\gamma$-secreting PBMCs were enumerated using Human IFN- $\gamma$ ELISPOT kit (cat no. DKW22-1000-096, Dakewe Biotech Co., Ltd., Beijing, China). Spots were counted using a Bioreader 5000 (Bio-Sys, Karben, Germany).

Assay for cytotoxic activity. Cytotoxic activity of the CTLs was examined based on the measurement of lactate dehydrogenase (LDH) release using a LDH cytotoxicity assay kit (Promega Corporation, Madison, WI, USA) according to the manufacturer's protocol. PBMCs $\left(5 \times 10^{6} / \mathrm{ml}\right)$ isolated from patients with LC and healthy donors were stimulated with rMBP-NAP $(0.2 \mathrm{mg} / \mathrm{ml})$ for 10 days, and used as the effector cells. EC-9706 and K562 were originally obtained from the Cell Bank of the Chinese Academy of Sciences (Shanghai, China) and were cultured in Iscove's modified Dulbecco's medium supplemented with $10 \%$ inactivated fetal calf serum (both from Thermo Fisher Scientific, Inc.) with $5 \% \mathrm{CO}_{2}$ at $37^{\circ} \mathrm{C}$, which were used as target cells $(16,17)$. EC-9706/K562 $\left(5 \times 10^{3}\right.$ cells/well) were co-cultured with various densities $\left(6.25 \times 10^{4}, 1.25 \times 10^{5}\right.$ and $\left.2.5 \times 10^{5}\right)$ of effector cells (PBMCs) at $37^{\circ} \mathrm{C}$ for $4 \mathrm{~h}$. The following effector: Target cell ratios were used: 12.5:1, 25:1 and 50:1. The measurement of LDH activity was analyzed by using the LDH assay kit. The ratio of cell toxicity (\% specific lysis) was calculated as follows: [(experimental release-effector spontaneous release-target spontaneous release)/(target maximum release-target spontaneous release)] x100.

Statistical analysis. All data are expressed as the mean \pm standard deviation. Differences between two groups were determined using an unpaired Student's t-test. One-way analysis of variance was used 
for multiple comparisons followed by a Bonferroni Comparison post hoc test. $\mathrm{P}<0.05$ was considered to indicate a statistically significant difference. All statistical analyses were performed by using software SPSS 10.0 (SPSS, Inc., Chicago, IL, USA).

\section{Results}

Patient enrollment. PBMCs samples from 12 lung patients were collected in the present study (Table I). Of these patients, $75 \%(9 / 12)$ were male and $25 \%(3 / 12)$ were female, and the median age was 61 years (range, $40-86$ years). In addition, $92 \%$ of patients $(11 / 12)$ were diagnosed with advanced stage disease (stage III-IV). Positive metastasis was observed in eight cases $(66.7 \%)$ and the middle stage of histological differentiation was detected in 10 cases $(83.3 \%)$.

Antitumor cytokine secretion of PBMCs is increased following rMBP-NAP stimulation. The secretion of antitumor cytokines from PBMCs in patients with LC and healthy donors, which were stimulated by rMBP-NAP, were measured using ELISA (Fig. 1). IFN- $\gamma$, IL-2 and TNF- $\alpha$ expression were significantly increased following rMBP-NAP treatment compared with the PBS control group.

Number of IFN- $\gamma$-producing PBMCs is increased following $r M B P-N A P$ stimulation. IFN- $\gamma$-producing cells serve an important role in antitumor immunotherapy (18). The number of IFN- $\gamma$-producing PBMCs in patients with LC and healthy donors, was measured using an ELISPOT assay (Fig. 2), and was demonstrated to be significantly increased following rMBP-NAP treatment compared with the PBS control group.

Cytolytic activity of PBMCs are increased following rMBP-NAP stimulation. The aforementioned results have demonstrated that antitumor cy tokine secretion and the number of IFN- $\gamma$-producing cells increased in PBMCs following rMBP-NAP stimulation. Additionally, the cytolytic activity was evaluated using a LDH release assay. EC-9706 and K562 cells were used as target cells, and the effector/target ratio were 12.5:1, 25:1 and 50:1. As shown in Fig. 3, PBMCs from patients with LC and healthy donors stimulated with rMBP-NAP, exhibited noticeable cytolytic activity at the effector/target ratio of 25:1 and 50:1. Following treatment with rMBP-NAP, the killing rates of PBMCs from health donors at 50:1 targeting EC-9706 and K562 cells were 24.9 and $29.6 \%$, respectively. The killing rates of PBMCs from patients with LC at 50:1 targeting EC-9706 and K562 cells were 26.3 and $31.9 \%$, respectively.

rMBP-NAP stimulates IL-12 mRNA expression though the TLR2 signaling pathway in PBMCs. The PBMCs from health donors and patients with LC were stimulated with rMBP-NAP, and the mRNA expression level of TLR 2 and IL-12 were examined by using RT-qPCR (Fig. 4). Following rMBP-NAP treatment, the levels of TLR2 and IL-12 mRNA were remarkably increased in PBMCs from health donors as well as patients with LC, compared with the PBS control groups. These results suggest that the upregulation of IL-12 mRNA expression by rMBP-NAP treatment was possibly mediated by the TLR2 signaling pathway in PBMCs.
Table I. Clinicopathological characteristics of patients with LC.

Characteristics No. of patients with LC

Sex

Female

3

Male

Age, years

$\geq 60 \quad 6$

$<60 \quad 6$

TNM stage

I 0

II 1

III-IV 11

Metastasis

Positive 4

Negative $\quad 8$

Histological differentiation

$\begin{array}{lr}\text { Low } & 2 \\ \text { Middle } & 10 \\ \text { High } & 0 \\ \text { Total } & 12\end{array}$

LC, lung cancer; TNM, tumor node metastasis.

\section{Discussion}

A number of cytokines have important immunomodulatory effects, and are used in tumor immunotherapy, including INF- $\gamma$, IL-2 and TNF- $\alpha(19,20)$. The US FDA has approved the use of INF- $\gamma$ and IL-2 as single agents for the clinical treatment of malignancies. Clinical trials of INF- $\gamma$ and IL-2 therapies in solid malignancies, such as bladder carcinoma and ovarian cancer, have met with varying degrees of success $(21,22)$. Several other cytokines, such as TNF- $\alpha$, have been demonstrated to exhibit antitumor efficacy against cancer, for example sarcoma and breast cancer $(23,24)$. ntTLR agonists have attracted increasing attention for its ability to promote the production of antitumor cytokines (e.g. IFN- $\gamma$, IL-2 and TNF- $\alpha$ ) in PBMCs (25). For example, $\mathrm{CpG}$-oligonucleotides ( $\mathrm{CpG}-\mathrm{ODN})$, which has been reported as an effective TLR9 agonist for antitumor treatment, may induce proliferative responses in PBMCs from patients with LC, accompanied by elevated cytokine secretion, including that of IFN- $\alpha$, IL-12 and TNF- $\alpha$ (26). BCG also induces Th1 cytokine production (IFN- $\gamma$, IL-12 and TNF- $\alpha$ ) of human PBMCs through the TLR4 signaling pathway in bladder carcinoma (27). Following treatment with rMBP-NAP, the secretion of INF- $\gamma$ from PBMCs, which serve important roles in antitumor treatment, was higher compared with that demonstrated by CpG-ODN in a previous study (26). The ability of rMBP-NAP, which induced antitumor cytokines production from the PBMCs, was lower compared with that of BCG. In our previous research, rMBP-NAP was observed to inhibit tumor growth by activating the TLR2 signaling pathway in murine models (14). Activated TLR2 induces 

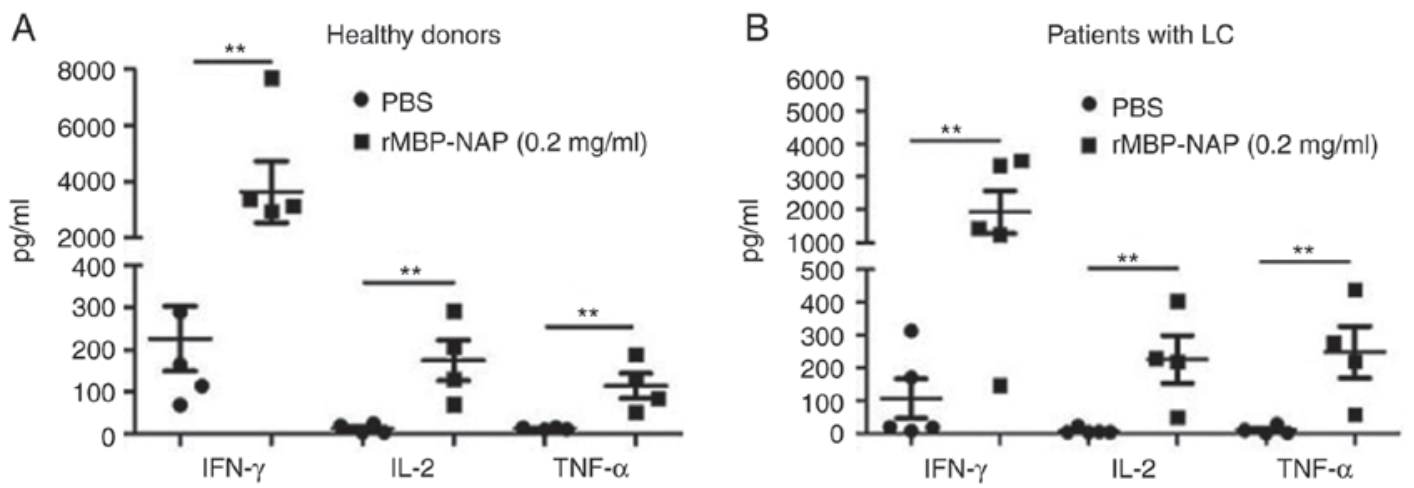

Figure 1. Expression levels of IFN- $\gamma$, IL-2 and TNF- $\alpha$ in PBMCs are upregulated following rMBP-NAP stimulation. PBMCs were stimulated with rMBP-NAP $(0.2 \mathrm{mg} / \mathrm{ml})$ for 5 days, and culture supernatants were collected for analysis of IFN- $\gamma$, IL-2 and TNF- $\alpha$ production in (A) healthy donors and (B) patients with LC. ${ }^{*} \mathrm{P}<0.01$, rMBP-NAP treatment vs. the PBS control. PBMCs were provided by five patients with LC and four healthy donors. rMBP-NAP, Recombinant Helicobacter pylori neutrophil-activating protein fused with maltose-binding protein; PBMCs, peripheral blood mononuclear cells; LC, lung cancer; IFN- $\gamma$, Interferon- $\gamma$; IL-2, interleukin-2; TNF- $\alpha$, tumor necrosis factor- $\alpha$.
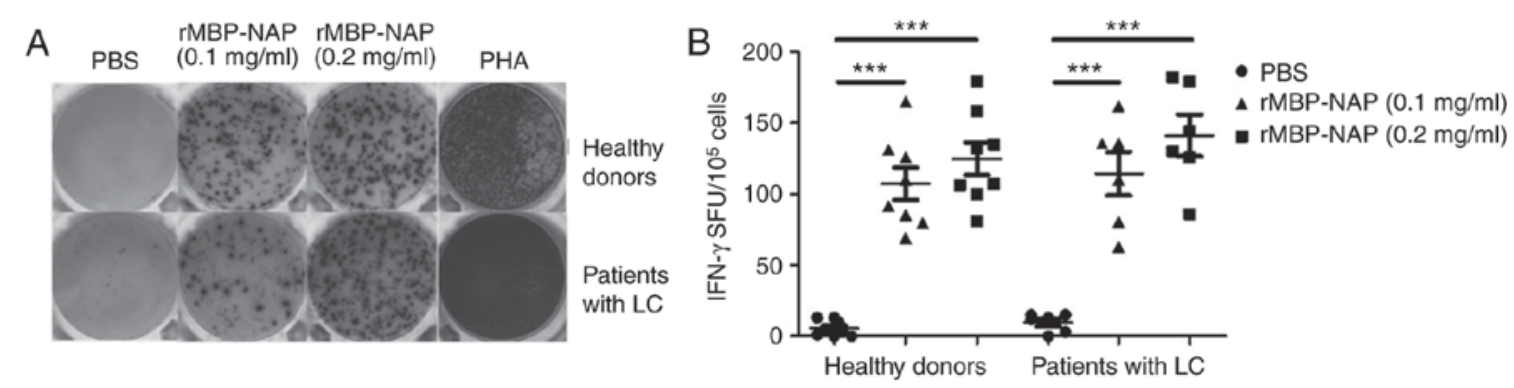

Figure 2. Number of IFN- $\gamma$-producing cells increases in PBMCs treated with rMBP-NAP. PBMCs from patients with LC and healthy donors were stimulated with 0.1 or $0.2 \mathrm{mg} / \mathrm{ml} \mathrm{rMBP-NAP} \mathrm{for} 5$ days. (A) An ELISPOT assay was performed in 96-well microtiter plates pre-coated with antibodies against IFN- $\gamma$. (B) Spots were counted using an ELISPOT plate reader. ${ }^{* * *} \mathrm{P}<0.001$, rMBP-NAP treatment vs. the PBS control. PBMCs were provided by six patients with LC and six healthy donors. ELISPOT, Enzyme-linked ImmunoSpot; rMBP-NAP, Recombinant Helicobacter pylori neutrophil-activating protein fused with maltose-binding protein; PBMCs, peripheral blood mononuclear cells; LC, lung cancer; PHA, phytohaemagglutinin; SFU, spot forming units.

the MyD88-dependent signaling pathway, which drives the activation of important pro-inflammatory transcription factors and subsequently promotes the production of Th1 cytokines (28). In the present study, the expression of TLR2 significantly increased following stimulation with rMBP-NAP, which indicated that the secretion of antitumor cytokines from PBMCs possibly depend on the TLR2 signaling pathway.

Cytotoxic lymphocytes, which serve an essential role in cancer immunotherapy, have been identified as $\mathrm{CD} 4^{+} \mathrm{CTL}$ and $\mathrm{CD}^{+} \mathrm{CTL}$ (29). The CD4 ${ }^{+}$CTLs produce multiple antitumor cytokines, including INF- $\gamma$, and facilitate $\mathrm{CD} 8^{+} \mathrm{T}$ cell activation and differentiation (30). CD8 ${ }^{+}$CTLs can directly kill tumor cells by releasing cytotoxic molecules, such as perforin and granzymes, and also possess the capacity to secreting INF- $\gamma$; thus, modulating the antitumor immune response $(31,32)$. Research has demonstrated the TLR agonists enhanced the ratio of IFN- $\gamma$-secreting cells, and the cytotoxicity of $\mathrm{CD}^{+}$and $\mathrm{CD} 8^{+}$CTLs in human PBMCs (33). It has been reported that the percentage of IFN- $\gamma$-secreting $\mathrm{CD}^{+}$ CTLs in PBMCs from patients with LC, was increased 6-folds following CpG-ODN stimulation, which was compared with PBS (26). In the present study, the number of IFN- $\gamma$-secreting CTLs from PBMCs stimulated with rMBP-NAP increased 10 fold in comparison with the PBS control. Furthermore, the cytolytic activity of PBMCs following rMBP-NAP treatment was evaluated. The cytolytic activity of PBMCs from patients with LC at 50:1 targeting EC-9706 and K562 cells was 3 fold higher compared with the PBS control, which is accordance with the 3 fold increase in the killing rate of PBMCs following BCG stimulation demonstrated in a previous study (34). These results suggest that rMBP-NAP effectively increased the number and the cytolytic ability of $\mathrm{CD} 4^{+}$and $\mathrm{CD} 8^{+}$ CTLs similar to that as the classical TLR agonists BCG and CpG-ODN.

In a conclusion, the results of the present study demonstrated that rMBP-NAP induced the production of multiple antitumor cytokines and enhanced CTL responses in PBMCs from patients with LC. Further investigations on the antitumor effect of rMBP-NAP are required to strengthen the evidence for clinical trial research.

\section{Acknowledgements}

Not applicable.

\section{Funding}

The present study was supported by the grants from the National Natural Science Foundation of China (grant nos. 81373119, 
A

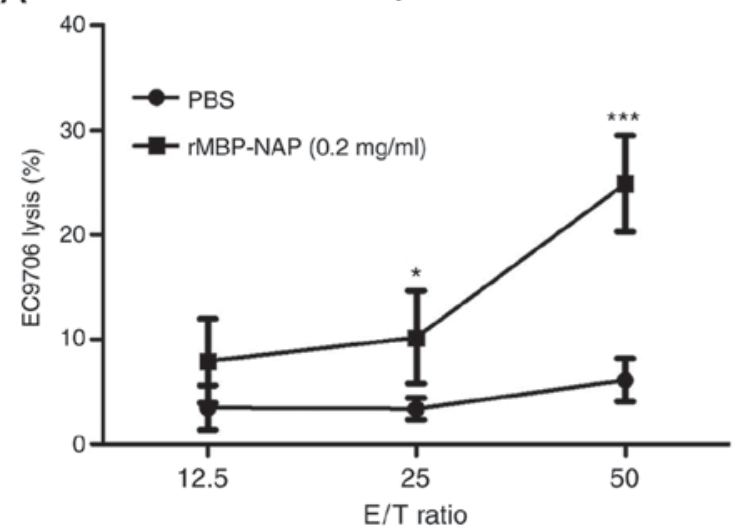

C

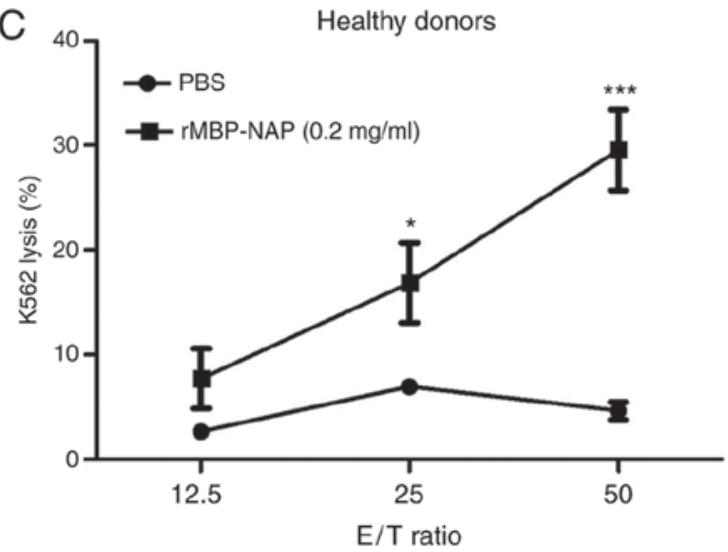

B

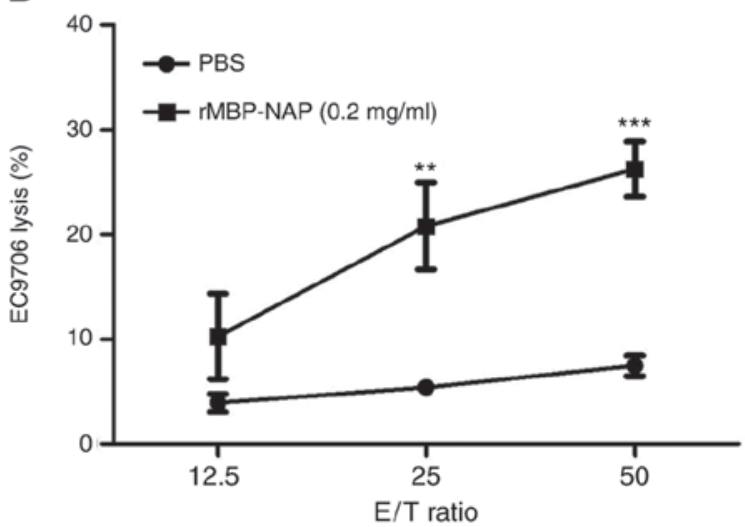

D

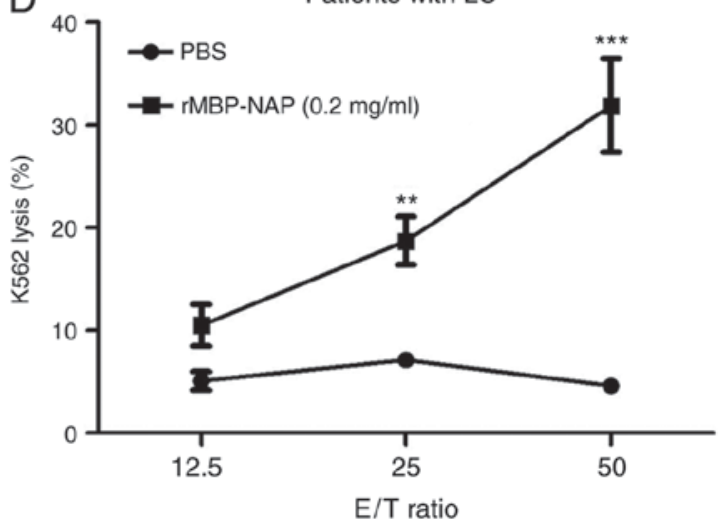

Figure 3. rMBP-NAP enhances the cytolytic activity of PBMCs. A lactate dehydrogenase release assay were performed to evaluate the cytolytic activity of PBMCs from health donor and patients with LC. PBMCs were stimulated with rMBP-NAP (0.2 mg/ml) for 10 days as effector cells. (A and B) EC-9706 and (C and D) K562 cells were used as target cells. The E/T ratios were 12.5:1, 25:1 and 50:1. ${ }^{*} \mathrm{P}<0.05,{ }^{* *} \mathrm{P}<0.01$ and ${ }^{* * * *} \mathrm{P}<0.001, \mathrm{rMBP}-\mathrm{NAP}$ treatment vs. the PBS control. PBMCs were provided by five patients with LC and five healthy donors. rMBP-NAP, Recombinant Helicobacter pylori neutrophil-activating protein fused with maltose-binding protein; PBMCs, peripheral blood mononuclear cells; LC, lung cancer; E/T, effector/target.
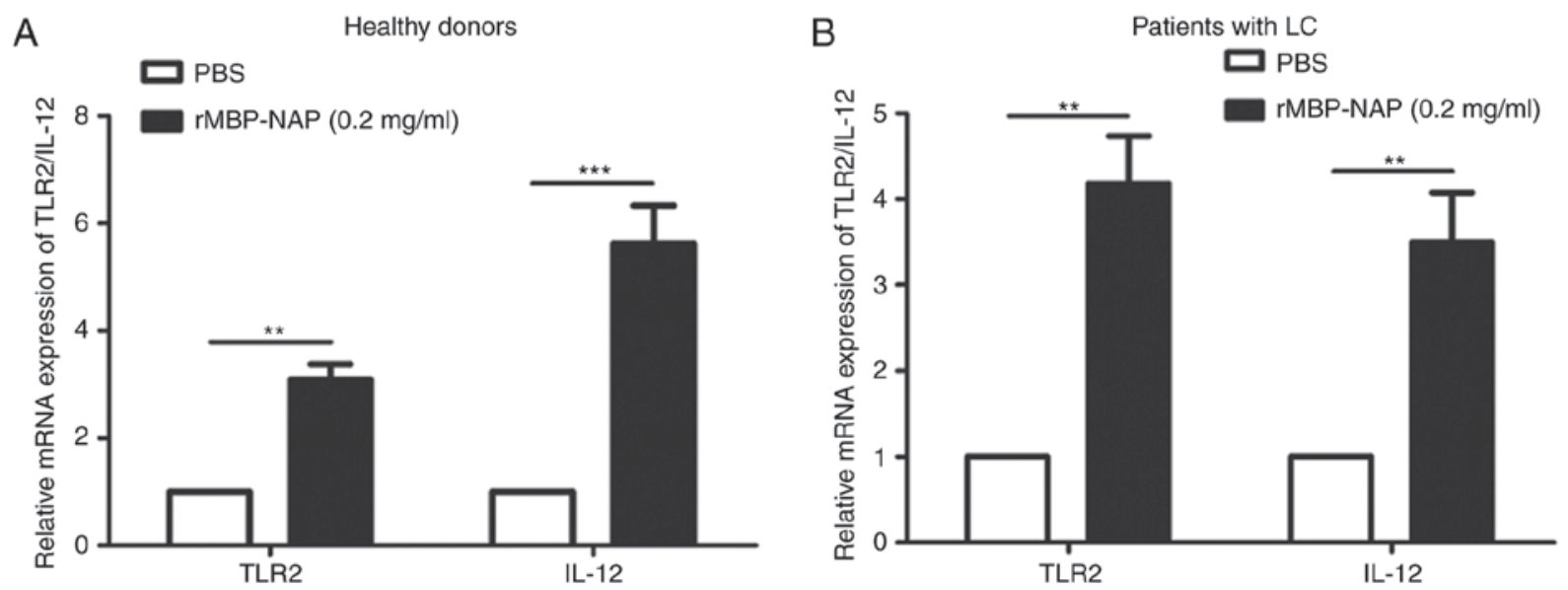

Figure 4. mRNA expression levels of TLR2 and IL-12 increase in PBMCs following rMBP-NAP stimulation. The mRNA relative expression of TLR2 for $16 \mathrm{~h}$ and IL-12 for $2 \mathrm{~h}$ stimulated with rMBP-NAP $(0.2 \mathrm{mg} / \mathrm{ml})$ in PBMCs from (A) healthy donors and (B) patients with LC was detected by reverse transcription-quantitative polymerase chain reaction. Data are expressed as the mean \pm standard deviation. ${ }^{* * *} \mathrm{P}<0.01$ and ${ }^{* * * *} \mathrm{P}<0.001$, rMBP-NAP treatment vs. the PBS control. PBMCs were provided by four patients with LC and four healthy donors. TLR2, Toll-like receptor 2; rMBP-NAP, Recombinant Helicobacter pylori neutrophil-activating protein fused with maltose-binding protein; PBMCs, peripheral blood mononuclear cells; LC, lung cancer; IL-12, interleukin-12.

81571526 and 81602537), Henan Provincial Science And Technology Innovation Outstanding Talent \& Distinguished Young Scholar Plan (grant nos. 184100510019 and 174200510025).

\section{Availability of data and materials}

All data generated or analyzed during this study are included in this published article. 


\section{Authors' contributions}

CD, LL, CZ, YZ, ZJ, TL and XG performed experiments, analyzed data, and reviewed the manuscript. XL and QK undertook project design and manuscript revisions.

\section{Ethics approval and consent to participate}

The present study was approved by the Ethical Committee of the First Affiliated Hospital of Zhengzhou University and study participants provided written informed consent.

\section{Patient consent for publication}

Written informed consent was obtained from each individual participant for the publication of their data.

\section{Competing interests}

The authors declare that they have no competing interests.

\section{References}

1. Adams S: Toll-like receptor agonists in cancer therapy. Immunother 1: 949-964, 2009.

2. Iwasaki A and Medzhitov R: Toll-like receptor control of the adaptive immune responses. Nat Immunol 5: 987-995, 2004.

3. Husek P, Pacovsky J, Chmelarova M, Podhola M and Brodak M: Methylation status as a predictor of intravesical Bacillus Calmette-Guérin (BCG) immunotherapy response of high grade non-muscle invasive bladder tumor. Biomed Pap Med Fac Univ Palacky Olomouc Czech Repub 161: 210-216, 2017.

4. Zhuang X, Wu T, Zhao Y, Hu X, Bao Y, Guo Y, Song Q, Li G, Tan S and Zhang Z: Lipid-enveloped zinc phosphate hybrid nanoparticles for codelivery of $\mathrm{H}-2 \mathrm{~K}(\mathrm{~b})$ and $\mathrm{H}-2 \mathrm{D}(\mathrm{b})$-restricted antigenic peptides and monophosphoryl lipid A to induce antitumor immunity against melanoma. J Control Release 228 26-37, 2016.

5. Cho JH, Lee HJ, Ko HJ, Yoon BI, Choe J, Kim KC, Hahn TW, Han JA, Choi SS, Jung YM, et al: The TLR7 agonist imiquimod induces anti-cancer effects via autophagic cell death and enhances anti-tumoral and systemic immunity during radiotherapy for melanoma. Oncotarget 8: 24932-24948, 2017

6. Guha M: Anticancer TLR agonists on the ropes. Nat Rev Drug Discov 11: 503-505, 2012.

7. Rakoff-Nahoum S and Medzhitov R: Toll-like receptors and cancer. Nat Rev Cancer 9: 57-63, 2009.

8. Shuang Chen, Wong MH, Schulte DJ, Arditi M and Michelsen KS: Differential expression of Toll-like receptor 2 (TLR2) and responses to TLR2 ligands between human and murine vascular endothelial cells. J Endotoxin Res 13: 281-296, 2007.

9. Santegoets SJ, Welters MJ and van der Burg SH: Monitoring of the immune dysfunction in cancer patients. Vaccines (Basel) 4 E9, 2016.

10. Kang QZ, Duan GC, Fan QT and Xi YL: Fusion expression of helicobacter pylori neutrophil-activating protein in E. coli. World J Gastroenterol 11: 454-456, 2005.

11. Fu HW: Helicobacter pylori neutrophil-activating protein: From molecular pathogenesis to clinical applications. World J Gastroenterol 20: 5294-5301, 2014.

12. Fang F, Ma J, Ni W, Wang F, Sun X, Li Y, Li Q, Xie F, Wang J, Zhai R, et al: $\mathrm{MUC1}$ and maltose binding protein recombinant fusion protein combined with Bacillus CalmetteGuerin induces MUC1specific and nonspecific antitumor immunity in mice. Mol Med Rep 10: 1056-1064, 2014.
13. Wang T, Liu X, Ji Z, Men Y, Du M, Ding C, Wu Y, Liu X and Kang Q: Antitumor and immunomodulatory effects of recombinant fusion protein rMBP-NAP through TLR-2 dependent mechanism in tumor bearing mice. Int Immunopharmacol 29: 876-883, 2015.

14. Wang T, Du M, Ji Z, Ding C, Wang C, Men Y, Liu S, Liang T, Liu X and Kang Q: Recombinant protein rMBP-NAP restricts tumor progression by triggering antitumor immunity in mouse metastatic lung cancer. Can J Physiol Pharmacol 9: 113-119, 2018

15. Livak KJ and Schmittgen TD: Analysis of relative gene expression data using real-time quantitative PCR and the $2-\Delta \Delta \mathrm{Ct}$ method. Methods 25: 402-408, 2001.

16. Liu QY, Zhou T, Zhao YY, Chen L, Gong MW, Xia QW, Ying MG, Zheng QH and Zhang QQ: Antitumor effects and related mechanisms of Penicitrinine A, a novel alkaloid with a unique spiro skeleton from the marine fungus penicillium citrinum. Mar Drugs 13: 4733-4753, 2015.

17. Horibe T, Kawamoto M, Kohno M and Kawakami K: Cytotoxic activity to acute myeloid leukemia cells by Antp-TPR hybrid peptide targeting Hsp90. J Biosci Bioeng 114: 96-103, 2012.

18. Motohashi S and Nakayama T: Clinical applications of natural killer T cell-based immunotherapy for cancer. Cancer Sci 99: 638-45, 2008.

19. Roychowdhury S and Caligiuri MA: Cytokine therapy for cancer: Antigen presentation. Cancer Treat Res 123: 49-266, 2005.

20. Baxevanis CN, Perez SA and Papamichail M: Cancer immunotherapy. Crit Rev Clin Lab Sci 46: 167-189, 2009.

21. Miller CH, Maher SG and Young HA: Clinical use of interferon-gamma. Ann N Y Acad Sci 1182: 69-79, 2009.

22. Minor DR, Moores SP and Chan JK: Prolonged survival after intraperitoneal interleukin-2 immunotherapy for recurrent ovarian cancer. Gynecol Oncol Rep 22: 43-44, 2017.

23. Jeon H, Kim D, Choi M, Kang S, Kim JY, Kim S and Jon S: Targeted cancer therapy using fusion protein of $\mathrm{TNF} \alpha$ and tumor-associated fibronectin-specific aptide. Mol Pharm 14: 3772-3779, 2017.

24. Wang K, Ren Y, Liu Y, Zhang J and He JJ: Tumor necrosis factor (TNF)- $\alpha$-induced protein 8-like-2 (TIPE2) inhibits proliferation and tumorigenesis in breast cancer cells. Oncol Res 25: 55-63, 2017.

25. Aranda F, Vacchelli E, Obrist F, Eggermont A, Galon J, Sautes-Fridman C, Cremer I, Henrik Ter Meulen J, Zitvogel L, Kroemer G and Galluzzi L: Trial watch: Toll-like receptor agonists in oncological indications. Oncoimmunology 3: e29179, 2014.

26. Ren T, Wen ZK, Liu ZM, Qian C, Liang YJ, Jin ML, Cai YY and Xu L: Targeting toll-like receptor 9 with CpG oligodeoxynucleotides enhances anti-tumor responses of peripheral blood mononuclear cells from human lung cancer patients. Cancer Invest 26: 448-55, 2008 .

27. Thanhäuser A, Böhle A, Flad HD, Ernst M, Mattern T and Ulmer AJ: Induction of bacillus-Calmette-Guérin-activated killer cells from human peripheral blood mononuclear cells against human bladder carcinoma cell lines in vitro. Cancer Immunol Immunother 37: 105-111, 1993.

28. De Nardo D: Toll-like receptors: Activation, signalling and transcriptional modulation. Cytokine 74: 181-189, 2015.

29. Bollard CM and Barrett AJ: CytotoxicT lymphocytes for leukemia and lymphoma. Hematology Am Soc Hematol Educ Program 2014: 565-569, 2014.

30. Martorelli D, Muraro E, Merlo A, Turrini R, Rosato A and Dolcetti R: Role of CD4+ cytotoxic T lymphocytes in the control of viral diseases and cancer. Int Rev Immunol 29: 371-402, 2010.

31. Weigelin B, Krause M and Friedl P: Cytotoxic T lymphocyte migration and effector function in the tumor microenvironment. Immunol Lett 138: 19-21, 2011.

32. Mempel TR and Bauer CA: Intravital imaging of CD8+ T cell function in cancer. Clin Exp Metastasis 26: 311-327, 2009.

33. Reynolds JM and Dong C: Toll-like receptor regulation of effector T lymphocyte function. Trends Immunol 34: 511-519, 2013.

34. Liu W, O'Donnell MA, Chen X, Han R and Luo Y: Recombinant bacillus Calmette-Guérin (BCG) expressing interferon-alpha $2 \mathrm{~B}$ enhances human mononuclear cell cytotoxicity against bladder cancer cell lines in vitro. Cancer Immunol Immunother 58: $1647-1655,2009$. 